Meta

Journal des traducteurs

Translators' Journal

\title{
Übersetzen als Sonderform des Risikomanagements
}

Wolfram Wilss

Volume 50, numéro 2, avril 2005

Processus et cheminements en traduction et interprétation

Processes and Pathways in Translation and Interpretation

URI : https://id.erudit.org/iderudit/011009ar

DOI : https://doi.org/10.7202/011009ar

Aller au sommaire du numéro

Éditeur(s)

Les Presses de l'Université de Montréal

ISSN

0026-0452 (imprimé)

1492-1421 (numérique)

Découvrir la revue

Citer cet article

Wilss, W. (2005). Übersetzen als Sonderform des Risikomanagements. Meta, 50(2), 656-664. https://doi.org/10.7202/011009ar

\section{Résumé de l'article}

Il n'est pas possible de dater l'entrée du mot « Risikomanagment » (RM) dans les dictionnaires de la langue allemande. Probablement, il s'agit d'un calque de l'expression américaine risk management, expression qui peut se vanter d'une longue tradition (Knight 1921) dans le monde anglo-américain. Pour approfondir une telle recherche dans le domaine de la langue allemande, il faudrait de nombreux dictionnaires mono- et plurilingues ainsi qu'une exégèse de la littérature spécialisée et exhaustive en sciences économiques. En d'autres termes, ceci nécessiterait un projet de recherche avec l'établissement d'un important corpus de textes avec l'infrastructure adéquate. Un seul homme - en l'occurrence un professeur émérite - ne peut y parvenir seul. 


\title{
Übersetzen als Sonderform des Risikomanagements
}

\author{
WOLFRAM WILSS \\ Universität des Saarlandes, Saarbrücken, Deutschland
}

\begin{abstract}
RÉSUMÉ
II n'est pas possible de dater l'entrée du mot «Risikomanagment» (RM) dans les dictionnaires de la langue allemande. Probablement, il s'agit d'un calque de l'expression américaine risk management, expression qui peut se vanter d'une longue tradition (Knight 1921) dans le monde anglo-américain. Pour approfondir une telle recherche dans le domaine de la langue allemande, il faudrait de nombreux dictionnaires mono- et plurilingues ainsi qu'une exégèse de la littérature spécialisée et exhaustive en sciences économiques. En d'autres termes, ceci nécessiterait un projet de recherche avec l'établissement d'un important corpus de textes avec l'infrastructure adéquate. Un seul homme - en l'occurrence un professeur émérite - ne peut y parvenir seul.
\end{abstract}

\begin{abstract}
We cannot date the first entry of the German word "Risikomanagment" (RM) in German dictionaries. Most probably, this is a calque of the American expression risk management, which has a long tradition in the Anglo-American world (Knight 1921). Should we want to make a thorough research in the German literature we would need not only numerous mono-and bilingual dictionaries, but would also have to go through the specialized literature of economic sciences. In other words, such an enterprise is not possible without establishing an important text corpus and the needed infrastructure. A one-man enterprise, which an emeritus represents nolens volens, can't possibly envisage such an enterprise.
\end{abstract}

\section{MOTS-CLÉS/KEYWORDS}

Risikofaktoren im Übersetzungsprozess, Vorlageabhängigkeit des Übersetzungsprozesses, Entscheidungsprozess, TAP

\section{Einleitung}

Wann das Wort „Risikomanagement“ (RM) im Lexikon der deutschen Sprache Fuß gefasst hat, lässt sich nicht mit Bestimmtheit sagen. Vermutlich ist es ein Lehnwort (eine Lehnübernahme) des amerikanischen „risk management“, das in Angloamerika schon eine längere Tradition hat (Knight 1921). Genaueres würden Recherchen in den einschlägigen ein- und mehrsprachigen Wörterbüchern in Verbindung mit der Auswertung der Fachliteratur der Betriebswirtschaftslehre (BWL) ergeben. Aber das wäre Sache eines corpuslinguistischen Forschungsprojekts mit den erforderlichen Personal- und Sachmitteln und nicht Sache eines Ein-Mann-Unternehmens, wie es ein Emeritus nolens volens betreibt.

Erwiesen ist zunächst nur, dass es sich bei RM um einen verhältnismäßig jungen Neologismus handelt, der lexikographisch noch nicht umfassend aufgearbeitet ist. Selbst ein so modernes Wörterbuch wie die 4., neu bearbeitete und erweiterte Auflage des DUDEN, Deutsches Universalwörterbuch (DUW 2001), das eine relativ große 
Anzahl an Einträgen mit „Management“ als Grundwort einer (i.d.R.) zweigliedrigen Zusammensetzung enthält, hat hier eine bemerkenswerte Lücke.

Diese Lücke ist umso auffälliger, als RM, wenn man die Tages- und Wochenpresse als Maßstab nimmt, in der letzten Dekade und den ersten Jahren des 21. Jahrhunderts ein allgegenwärtiges, vielfach verwendbares (multifunktionales) und somit bequem handhabbares Schlagwort geworden ist. Da ist einmal die Reaktorkatastrophe von Tschernobyl (1986) mit ihren bis heute unbewältigten verheerenden Folgen für die Weltgesellschaft, zum andern das 1986 von dem Münchener Soziologen Ulrich Beck veröffentlichte, in viele Sprachen übersetzte Buch „Risikogesellschaft“, und schließlich die Tatsache, dass wir in einer von Risiken umstellten Zeit leben, die immer mehr Risiko/Krisenmanagement erfordert. Becks Buch wurde ein globaler Bestseller, nicht nur, weil er für seine „Botschaft“ einen griffigen Titel erfand, sondern auch, weil er innerhalb und außerhalb Deutschlands der Gesellschaft ein Risikobewußtsein einimpfte, das heute gleichermaßen auf politischem, wirtschaftlichem (wirtschaftspolitischem), technischem, natur-/wissenschaftlichem, militärischem und soziokulturellem Gebiet manifest ist. Es signalisiert ein Nebeneinander und Übereinander von „Bedrohungsszenarios“, deren kombinierte Wucht alles bisher Dagewesene bei weitem übertrifft (Bahro/Strand 2002; dort weitere Lit.).

RM hat sich in fast allen Fugen unserer Lebens- und Wissenschaftspraxis festgesetzt. Es überwölbt - zusammen mit „Krisenmanagement“ - ungebremst alle Formen menschlicher Tätigkeit und menschlicher Befindlichkeiten, vom AIDS/ BSE-Risikofaktor, dem Vabanquespiel mit dem technischen/gentechnischen Risiko, dem Globalisierungsrisiko, dem Risikoinventar in der „New Economy“, dem Suchtmittelrisiko, den „fatty junkies“, für die „das große Fressen“ der maßgebende Lebensinhalt ist, bis hin zur Risikominderung/Risokominimierung in der Luftfahrt („Jede Woche eine Flugzeugkatastrophe?" NZZ 6.8.1998, 37) oder den Risikofaktoren des letztlich unberechenbaren Fortschritts und des Beschleunigungswahnsinns (z.B. im Alpentransit).

All das (und vieles mehr) ist ein Indiz dafür, dass RM ein höchst ambivalenter Fluchtpunkt des Denkens und Handelns der modernen Gesellschaft ist, der eine bestimmte Sehweise auf bestehende Verhältnisse erlaubt: In seinen vielen Erscheinungsformen ist RM ein fester Bestandteil unsicherheitsgeleiteter Entscheidungsprozesse, die unsere auf Profit und Optimierung fixierte, modernisierungs- und informationssüchtige Produktions- und Konsumgesellschaft tagtäglich praktiziert.

Des Weiteren verweist RM auf das unaufhaltsame Vordringen quantitativer Denk- und Handlungsweisen. Diese sind für eine verwertungs- und evaluierungsorientierte Gesellschaft typisch: Man sieht in RM eine, wenn nicht d i e Möglichkeit, politische, wirtschaftliche und vor allem technische Großprojekte schneller, billiger und flächendeckender als bisher durchzuführen, auch wenn im Rahmen dieser Hyper/ Cyber-Aktivität qualitative Abstriche in Kauf genommen werden müssen und auch in Kauf genommen werden.

\section{Risikofaktoren im Übersetzungsprozess}

Übersetzen ist durch spezifische Tätigkeitsumstände und Tätigkeitsvoraussetzungen geprägt. Der wichtigste Tätigkeitsumstand - und gleichzeitig der dominierende Risikofaktor - ist die prinzipielle Vorlageabhängigkeit des Übersetzungsprozesses: Wo 
kein Ausgangstext, da auch keine Übersetzung. Der Übersetzer kann sich sein Tun nicht selbst zuschreiben; er steht nicht im Kontext einer unmittelbaren, sondern einer vermittelnden Kommunikation, in welcher er im Rahmen verbindlicher text(typ)spezifischer und empfängerspezifischer Bedingungen auf der Grundlage der ihm zur Verfügung stehenden Wissensbestände reaktiv antwortet.

Dieser reproduktive Charakter des Übersetzens mag vordergründig als ein Vorteil erscheinen, weil es der Übersetzer mit „vorgedachten“ und „vorformulierten“ Texten zu tun hat. Übersetzen gilt als ein Teil der allgemeinen Informationsverarbeitungskapazität und Informationsverarbeitungspraxis des Menschen, in der „Wissen für wen?, wozu?, Wissen was?, Wissen wieso?“ zusammenwirken (müssen), dass der Übersetzer darauf einen interlingualen/interkulturellen Wissenstransfer aufbauen kann.

Indes, der vermeintliche Vorteil wird durch die Fremdbestimmtheit übersetzerischer Tätigkeit aufgehoben, und zwar aus zwei Gründen: Erstens sind Sprachen und Kulturen nur sehr begrenzt auf der Basis von Eins-zu-Eins-Entsprechungen (wörtliche Übersetzung) aufeinander abbildbar. (Die mageren Ergebnisse der maschinellen (nicht-interaktiven) Übersetzung sprechen hier eine deutliche Sprache; Wilss 1988). Deshalb muss der Übersetzer praktisch unablässig versuchen, RMabwägende Kompensationsstrategien zu entwickeln, die, wie die Berufspraxis zeigt, in immer neuen Formen und Gewichtungen in Erscheinung treten. Sie zwingen den Übersetzer oft zu komplexen zielsprachlichen Textmanipulationen, wenn er wenigstens auf die Ebene der „Suboptimalität“ gelangen will (zu Kompensationsstrategien vgl. Wilss 1992, Kap. III; zur Differenzierung zwischen „Optimalität“ und „Suboptimalität“ vgl. Wilss 2000, Kap. 12.3).

Zweitens gibt es für die Vermittlungsleistung des Übersetzers, von interlingual hochstandardisierten Texten abgesehen, keinen Standardinput (und infolgedessen auch keinen Standardoutput, übrigens ein Gesichtspunkt, der die prinzipiellen Schwierigkeiten der soeben erwähnten maschinellen Übersetzung zeigt). Der professionelle Übersetzer muss tagtäglich damit rechnen, dass er mit Texten konfrontiert wird, deren Übersetzung stets neue Bemühungen um die Erkenntnis des Fremdpsychischen, neue (nicht nur oder nur z.T. generalisierbare) Strategieentscheidungen, neue Formen der (prospektiven) Risikominderung und des (retrospektiven) Krisenmanagements und neue Äquivalenzüberlegungen verlangt, und dies oft in einem von kommunikativen Sachzwängen diktierten, unangemessenen Tempo.

Diese Zusammenhänge werden von der Öffentlichkeit im Allgemeinen nicht oder nur widerwillig zur Kenntnis genommen, obwohl der Umfang übersetzerischer Tätigkeit in den letzten Jahrzehnten im Zuge der fortschreitenden Internationalisierung der (Fach-)Kommunikation zugenommen hat (Wilss 2003). Von Ausnahmen abgesehen, z.B. der kontroversen Diskussion über die Qualität der deutschen Übersetzung von „Lemprière’s Dictionary“ von Lawrence Norfolk (Richter 1993; GerzymischArbogast 1994; Horton 1994), verhält sich die Öffentlichkeit der Tätigkeit des Übersetzers gegenüber weithin indifferent, möglicherweise ein Grund, warum die Übersetzungswissenschaft (ÜW) bisher darauf verzichtet hat, sich an der intensiven, von der BWL ausgehenden RM-Diskussion zu beteiligen.

Die Öffentlichkeit hat im Allgemeinen keine differenzierten Vorstellungen von der mentalen Tätigkeit des Übersetzers. Noch weniger kann/will sie verstehen, dass Übersetzen ein weithin risikobesetztes Unterfangen ist, da sich der Übersetzer ständig 
mit zwei Sprachen und zwei Kulturen herumschlagen muss, wo schon die Beschäftigung mit einer Sprache und einer Kultur, wie PISA gezeigt hat, hinlänglich Probleme aufwirft, die man nicht routinemäßig aus der Welt schaffen kann.

Während man von einem Wissenschaftler klare und plausible Sachargumentation, von einem Künstler Phantasie und Kreativität, von einem Arzt Sachkenntnis und Einfühlungsvermögen, von einem Pfarrer Hilfsbereitschaft und seelischen Beistand erwartet, ist das Bild des Übersetzers in den Augen der Öffentlichkeit verschwommen. Das so ungefähr einzige, worin in der Öffentlichkeit Konsens besteht, ist, dass in den meisten Fällen (jedenfalls in der Fachkommunikation) eine „Informativübersetzung" ohne jeden stilistischen Anspruch genügt, dass der Übersetzer möglichst unauffällig (d.h. unter anderem möglichst ohne unangenehme Fragen an den Ausgangstextautor zu stellen), möglichst schnell und möglichst billig arbeitet. In den Augen der Öffentlichkeit ist der Übersetzer „der Experte fürs Allgemeine“, der in den gelegentlichen, auf Meinungsumfragen beruhenden „Berufs-Ratings“ („BerufsRankings“), vom Arzt über den Professor und den Pfarrer bis hin zum Politiker und Gebrauchtwarenhändler, keinerlei Erwähnung findet.

Für den Kunden ist der Übersetzer kein Innovator, sondern ein „Nachläufer“, der ihm ein möglichst fehlerfreies Produkt liefert, mit dem ersterer die Vorstellung eines Produktmehrwerts verbindet, weil er mit dem Originaltext mangels Fremdsprachenund Fremdkultur-Kenntnissen nichts anzufangen weiß und deshalb auf die Unterstützung durch den Übersetzer im Rahmen einer „linguistic support“-Konzeption angewiesen ist, die sich geringer Wertschätzung erfreut:

The Bureau of the Census, Department of Commerce, Immigration and Naturalization Service and other agencies have tended to categorize translators and interpreters as non-professionals... (Hammond 1994,2).

Auf den Nenner gebracht, könnte man sagen, dass der Übersetzer mit einer nicht zu ändernden Situation konfrontiert ist, die durch die bedrängende Macht eines bereits ausgestalteten Textes charakterisiert ist, in dem sprachliche, epistemische, kulturelle, gesellschaftliche und individuelle Faktoren zusammenwirken, die alle mehr oder minder RM-relevant sind. Zusammengenommen ergeben sie ein komplexes RMSzenario, in dem es keine festen, einzeltextübergreifenden RM-Normen, -Kategorien und -Methoden gibt.

Jede Übersetzung ist sozusagen ein Ritt über den Bodensee, und wenn der Übersetzer einmal glücklich das rettende Ufer erreicht hat, bedeutet dies nicht, dass er für zukünftige Ritte genügend Vorsorge getroffen hat. Die Frontlinien zwischen Auftraggeber und Übersetzer - die neuerdings intensiv diskutierte „client-/server relationship“- verändert sich ständig. Mit Ausnahme der relativ wenigen Übersetzer, die es sich leisten können, sich auf einem thematisch und sprachlich eng begrenzten Terrain zu betätigen oder mit einem einzigen Autor (z.B. im Rahmen der vom Verlag Luchterhand finanzierten Seminare zwischen Günter Grass und seinen Übersetzern) zusammenzuarbeiten, gibt es für den Übersetzer, jedenfalls den Nicht-Routinier, keinen einigermaßen berechenbaren „Erwartungshorizont“ oder kalkulierbare „Vorausfestlegungen“ oder „interaktive Möglichkeiten“. Deshalb bleibt oft nur wenig oder gar kein Raum für übersetzerische Selbstbestimmung, für detaillierte Analyseoperationen und für unter Umständen langwierige Prozesse der Bewusstmachung des jeweils zu investierenden RM-Aufwands. 
Bei allem Bemühen um Perfektion (d.h. Risikominderung bis zum Zero-Punkt) ist Perfektionismus für Übersetzer kein realistischer Orientierungspunkt. Dies ist natürlich in einem Zeitalter, in dem sich Perfektionismus (Optimierung) auf wissenschaftlichem, technischem und, nicht zuletzt, auf sportlichem Gebiet größter Wertschätzung erfreut, und in dem kaum noch jemand sich mit weniger als dem Perfektiven (dem technischen Non-plus-ultra) begnügen will, ein gravierendes Handicap, das dem in der Regel ohnedies schwachen „Selbstkonzept" des Übersetzers Abbruch tut. Er ist gezwungen, in einem Milieu zu arbeiten. in dem das sog. „Restrisiko" sein ständiger Begleiter ist.

Wer einmal aufgrund eigener Erfahrung Übersetzen als das komplexe Spiel nicht bis ins letzte Detail rational aufzuhellender Kräfte und Gegenkräfte erlebt hat, erkennt die Begrenztheit der eigenen Möglichkeiten als das konstitutive Prinzip des Übersetzens und wird gegenüber lautstarken, aber nicht (immer) überzeugend vorgetragenen Argumenten, alles Übersetzen ließe sich auf ein universales funktionales Grundtheorem reduzieren, mit Skepsis reagieren. Für den praktizierenden Übersetzer steht immer mehr auf dem Spiel als für den Übersetzungstheoretiker, der die verwickelten übersetzerischen Zusammenhänge nur monokausal durchdenkt und den offenen Horizont übersetzerischer Dispositionen und Wertvorstellungen seinem theoretischen Unifizierungspostulat unterordnet. Übersetzen ist die Manifestation einer operativen Intelligenz, die dann besonders aktiv wird, wenn sich Übersetzen als Entscheidungsprozess präsentiert.

\section{3. Übersetzen als Entscheidungsprozess}

Die Fixiertheit auf eine Schreibsituation, die keine (oder nur eine geringe) eigene Makroplanung im Sinne einer von einem Vortext unabhängigen Schreibstrategie erfordert, ist wahrscheinlich die Ursache dafür, dass der Begriff „Entscheidungsprozess" in der übersetzungswissenschaftlichen Fachliteratur vage ist, und dass es, streng genommen, bis heute keine umfassende Monographie über das Übersetzen als Entscheidungsprozess gibt. Entscheidungstheorie und Entscheidungsforschung, zwei in den letzten drei Jahrzehnten mächtig ins Kraut geschossene Teilgebiete der BWLbestimmten Psychologie, sind in der ÜW noch weithin terra incognita. Vor allem die beiden Begriffe „Entscheidung unter Sicherheit“ und „Entscheidung unter Unsicherheit" (Kirchler 1999) müssen auf ihren übersetzungsmethodischen Aufschlusswert hin überprüft werden.

\subsection{Entscheidung unter Sicherheit}

Jeder Übersetzer weiß, dass er ständig in Situationen gerät, in dem er zwischen mindestens zwei „Optionen“ eine Entscheidung treffen muss:

Wenn Entscheidungsträger vollständige Informationen über die zählbaren Alternativen und Sicherheit über deren Konsequenzen besitzen, werden Entscheidungen unter Sicherheit getroffen. Wenn völlige Klarheit über alle Entscheidungsmöglichkeiten und Ergebnisse besteht, Personen unendlich sensibel sind, was die Unterschiede zwischen den Entscheidungsmöglichkeiten anbelangt und deshalb alle Alternativen nach subjektiven Präferenzen reichen können, dann werden Entscheidungen unter Sicherheit gefällt. Häufig besteht bezüglich der Konsequenzen von Entscheidungsalternativen 
jedoch Unsicherheit. Die theoretischen Überlegungen und empirischen Studien über Entscheidungen differieren je nachdem, ob Entscheidungen unter Gewissheit, Risiko, Ambiguität oder Unwissenheit gefällt werden ... In diesem Fall können subjektive Wahrscheinlichkeiten erhoben werden, mit denen Ereignisse und Konsequenzen erwartet werden. Risikoentscheidungen sind also Entscheidungen über Alternativen und Konsequenzen, welche mit einer definierten Wahrscheinlichkeit eintreten können (Kirchler 1999, 23f.).

Dafür ein einfaches Beispiel:

(1) Having finished his job, he went home.

Diese Äußerung können wir als Satzgefüge mit vorangestellter, nichteingeleiteter Partizipialkonstruktion (PK) und angeschlossenem Hauptsatz (Bezugssatz) beschreiben, und wir wissen, dass es sich dabei um eine im Englischen äußerst beliebte, alltagssprachliche, literatursprachliche und fachsprachliche Syntaxkonfiguration handelt (Wilss 1972). Wir können (ohne nennenswerte Sinnänderung) die Position der beiden Teilsätze vertauschen:

(2) He went home, having finished his job.

Vermutlich wäre ein englischer Muttersprachler damit nicht ganz zufrieden, weil er bei dieser Teilsatzabfolge eine Einleitung mit "after" (oder "since") erwartet:

(3) He went home, after having finished his job.

Aber auch nach dieser Änderung würde er wahrscheinlich der Teilsatzabfolge

(4) After having finished his job (after finishing his job), he went home.

den Vorzug geben (nebenbei bemerkt, ein gutes Beispiel für die psychologische Realität der Grammatik: Zuerst beendet besagte Person ihren Job, dann geht sie nach Hause).

Nun zur Übersetzung ins Deutsche: Hier gibt es drei Standardvarianten, die man zusammen als „Übersetzungsparadigma“ bezeichnen kann:

(5) Er beendete seinen Job und ging dann nach Hause (parataktische Lösung)

(6) Nachdem er seinen Job beendet hatte, ging er nach Hause

(hypotaktische Lösung)

(7) Nach Beendigung (Erledigung) seines Jobs ging er nach Hause

(Nominalisierung).

Eine syntaktische Eins-zu-Eins-Besprechung (wörtliche Übersetzung) ist gebrauchsnormativ nicht zulässig (obwohl nach den Regeln des deutschen Grammatiksystems zulässig), es sei denn, der Sprecher will den betreffenden Sachverhalt, etwa im Stil der englischen Filserbriefe (Daum 1990) absichtlich verfremden oder ironisieren:

(8) Seine Arbeit beendet habend, ging er nach Hause.

Festzuhalten ist, dass das, was hier unter Sicherheit stattfindet, RM-irrelevant ist. Der Übersetzer braucht keine langen Überlegungen darüber anzustellen, welche Variante er wählen oder, wie es im Neuestdeutsch heißt, „präferieren“ soll. Alle drei Varianten kennt der in der Ausgangs- und der Zielsprache einigermaßen vertraute Übersetzer. Er kann sich also wie eine Art „Zufallsgenerator“ verhalten - mit einer 
kleinen Einschränkung: Es kann sein, dass er aus makrokontextuellen (makrostilistischen) Gründen einer Variante bewusst den Vorzug gibt. In einem Polizeiprotokoll ist (7) eher erwartbar als (5) oder (6). Entscheidend ist, dass der Übersetzer eine Entscheidung treffen $\mathrm{m}$ u s $\mathrm{s}$.

Dazu Jungermann:

Mit dem Begriff „Entscheidung“ verbinden wir im allgemeinen mehr oder weniger überlegtes, konfliktbewusstes, abwägendes und zielorientiertes Handeln ... oft werden Entscheidungen auch rasch und ohne längeres Nachdenken getroffen, wenn beispielsweise das Problem trivial ist und es daher einer längeren Beschäftigung nicht lohnt oder wenn das Problem gut bekannt ist und seine Lösung schon Gewohnheit oder Routine geworden ist $(1998,3)$.

\subsection{Entscheidung unter Unsicherheit}

Von welchen Faktoren ein komplexer übersetzerischer Entscheidungsprozess abhängt, ist von Text zu Text und von Übersetzer zu Übersetzer (Übersetzungskontext) verschieden. Es gibt keine allgemeine, universell applizierbare Entscheidungskompetenz, genauso wenig wie es eine allgemeine, universell applizierbare Kreativitätskompetenz gibt. Wer in seinem Wissen unsicher ist, kann auch nicht sicher wissen, wie er entscheiden soll. Hat sich ein Übersetzer in einer komplexen Situation für eine seines Erachtens erfolgversprechende Übersetzungsprozedur entschieden, setzt er in der Regel nicht nur e i n e n Entscheidungsprozess, sondern eine Kette von wechselseitig abhängigen Entscheidungsprozessen in Gang. Eine solche Entscheidungskette, für die Neubert die Formulierung "optimisation of the translator's decision process“ $(1984,61)$ gefunden hat, schränkt seinen Verhaltensspielraum progressiv ein: Wenn a, dann b, wenn b, dann c usw. Entscheidungsketten kann man sich in Form eines links/rechtsgerichteten Entscheidungsbaums vorstellen, der durch das Prinzip der abnehmenden Kumulation gekennzeichnet ist.

Allerdings sind in der Übersetzungspraxis (und damit auch im Übersetzungsunterricht, Wilss 1996) die einzelnen Entscheidungsprozesse (Inferenzprozesse) längst nicht immer so übersichtlich wie in dem hier vorgeführten Fall. Je komplexer die syntaktisch-lexikalischen Verhältnisse (nicht zu vergessen das soziokulturelle „embedding“) sind, desto komplexer ist auch die Entscheidungsproblematik (Levy 1981).

Die Diskussion von Übungsklausuren in Saarbrücken hat gezeigt, dass die Plausibität von Entscheidungen ganz unterschiedlichen Gesichtspunkten unterlag. Entscheidungsprozesse sind „relative“ Ereignisse, wobei die Realitivität dort ihre Grenzen findet, wo ein Entscheidungsprozess in subjektive Willkür (Dezisionismus) ausartet. Dies ist vor allem dann zu beobachten, wenn ein Unsicherheitsfaktor - oder ein Bündel von Unsicherheitsfaktoren - ein besonders hohes Maß an übersetzerischer Hilflosigkeit verrät.

Ein anderer Fall liegt vor, wenn ein Übersetzer klüger zu sein glaubt als der Ausgangstextautor. Allerdings kommt es vor, dass ein Übersetzer klüger sein $\mathrm{m}$ u s s als der Ausgangstextautor, dann nämlich, wenn evident ist, dass er miserabel formulierte Ausgangstexte - unter Umständen mit einem hohen RM-Aufwand - erst einmal „reparieren“ muss, bevor er mit dem Transfer beginnen kann (intralinguale/ausgangssprachliche Übersetzung/Paraphrase als Vorstufe zum interlingualen Transfer). 


\section{Schlussbemerkungen}

Was sich alles an mentalen Prozessen abspielt, wenn sich ein Übersetzer entscheidungsbewusst verhält, ist bisher kaum ansatzweise aufgeklärt. Hier kann die von Krings diskutierte, aber noch nicht operationalisierte Methode des Lauten Denkens $($ der „Thinking-Aloud Protocols $=$ TAPs) hilfreich sein (1986). Vorrangig kommt es darauf an, die Entscheidungskompetenz des Übersetzers unter dem Aspekt der übersetzerischen Handlungsbedingungen (Termindruck etc.: Nord 1988; Wilss 2000), der übersetzerischen Handlungsdisponibilitäten und, nicht zuletzt, des zumutbaren Schwierigkeitsgrads des zu übersetzenden Textes zu untersuchen und didaktisch zu implementieren.

Von Entscheidungen ist heute im diskursiven und im algorithmischen (binären) Zusammenhang die Rede. Die ÜW, sofern sie an Entscheidungsfragen interessiert ist, muss sich folglich auch mit dem Entscheidungsbegriff in der Computerlinguistik und der Spieltheorie auseinandersetzen, gleichgültig, ob sich das mit ihrem traditionellen Selbstverständnis als anthropologische Wissenschaft verträgt oder nicht. Selbstverständlich wird die Erforschung von Entscheidungsprozessen innerhalb und außerhalb des RM-Kontextes nie eine ganz exakte (nomologische) Wissenschaft werden; sie kann sich nicht an radikale, naturalistisch vorgegebene Verfahrensweisen ausliefern, sondern sie braucht heuristische Methoden für die übersetzerische Entscheidungsfindung. Wenn sie so vorgeht, könnte das dazu beitragen, dass die ÜW ihr Dilemma, mehr Anspruch als Wirklichkeit zu sein, überwindet und dass ein komplexer, die Psychologie als Leitwissenschaft integrierender Begriff von übersetzungsbezogener Risikoforschung entsteht, die disziplinär und transdisziplinär Entscheidungspsychologie und Wissenspsychologie miteinander verbindet und zwischen Entscheidung unter Sicherheit und Entscheidung unter Unsicherheit differenziert.

\section{RÉFÉRENCES}

Bahro, M./Strand, J. (2002): Die Wahrnehmung von Risiken, in Schweizer Monatshefte 9/82, Sept. 2002, p. 30-35.

Beck, U. (1986): Risikogesellschaft, Auf dem Weg in eine andere Moderne, Frankfurt/M., Suhrkamp.

Daum, E. (1990): Higheightingsfull Your True Gisela, Neue Filserbriefe aus der Süddeutschen Zeitung, Frankfurt/M., Eichborn.

Gerzymisch-Arbogast, H. (1994): Übersetzungswissenschaftliches Propädeutikum, Tübingen, Francke.

Hammond, D.L. (1994): (ed.): Professional Issus for Translators and Interpreters, Editor's Remark, Amsterdam/Philodelphia, John Benjamins, p. 1-10.

Horton, D. (1994): Literary Translation between Equivalence and Manipulation, Lawrence Norfolk's Lemprièrés Dictionary in German, in Neophilologus 78, p. 175-194.

Jungermann, H. et al. (1998): Die Psychologie der Entscheidung. Eine Einführung. Heidelberg, Spektrum.

Kirchler, E. M. (1999): Wirtschaftspsychologie, Grundlagen und Anwendungsfelder der Ökonomischen Psychologie. Göttingen, Hogrefe.

KNight, F. H. (1921), Risk, uncertainty and profit. Boston/New York: Houghton \% Mifflin.

Krings, H.P. (1986): Was in den Köpfen von Übersetzern vorgeht, Eine empirische Untersuchung zur Struktur des Übersetzungsprozesses an fortgeschrittenen Frönzösischlernern, Tübingen, Narr.

Levy, J. (1981): Übersetzung als Entscheidungsprozess, in W. WiLss (Hg.), Übersetzungswissenschaft, Darmstadt: Wissenschaftliche Buchgesellschaft, p. 219-235. 
Neubert, A. (1984): Translation studies and applied linguistics, in AILA Review No. 1, p. 49-64.

Nord, C. (1988): Textanalyse und Übersetzen, Heidelberg: Groos.

RichteR, W. (1993): Tausend nach Ratten geworfen, Übersetzerstreit um Lemprières Wörterbuch, in Lebende Sprachen XXXVIII/3, p. 97-100.

Wilss, W. (1971): Englische Partizipialkonstruktionen und ihre Wiedergabe im Deutschen, in Bausch, K.-R./Gauger, H.-M. (Hg.), Interlinguistica, Sprachvergleich und Übersetzung, Festschrift zum 60, Geburtstag von Mario Wandruszka, Tübingen, Niemeyer, p. 555-568.

WiLss, W. (1988): Kognition und Übersetzen, Zu Theorie und Praxis der menschlichen und der maschinellen Übersetzung, Tübingen, Niemeyer.

WiLss, W. (1992): Übersetzungsfertigkeit, Annäherungen an einen komplexen übersetzungspraktischen Begriff, Tübingen, Narr.

WiLss, W. (1996): Übersetzungsunterricht, Eine Einführung, Tübingen: Narr.

WiLss, W. (2000): Wandlungen eines Universitätsinstituts, Vom „Dolmetscherinstitut“ zur „Fachrichtung Angewandte Sprachwissenschaft sowie Übersetzen und Dolmetschen der Universität des Saarlandes, St. Ingbert, Röhrig Universitätsverlag.

Wilss, W. (Hg.): Die Zukunft der internationalen Kommunikation im 21, Jahrhundert (20012020), Tübingen, Narr. 